\title{
Swerving Orientation of Spin-Stabilized Projectile for Fixed-Cant Canard Control Input
}

\author{
Xu-dong Liu, Dong-guang Li, and Qiang Shen \\ School of Mechatronical Engineering, Beijing Institute of Technology, Beijing 10081, China \\ Correspondence should be addressed to Dong-guang Li; lidongguang@bit.edu.cn
}

Received 14 December 2014; Accepted 2 March 2015

Academic Editor: Hakim Naceur

Copyright (C) $2015 \mathrm{Xu}$-dong Liu et al. This is an open access article distributed under the Creative Commons Attribution License, which permits unrestricted use, distribution, and reproduction in any medium, provided the original work is properly cited.

\begin{abstract}
Due to the large launch overload and high spin rate of spin-stabilized projectile, no attitude sensor is adopted in square crossing fixed-cant canard concept, which causes the lack of existing projectile linear theory for the close form solution of swerving motion. This work focuses on swerving orientation prediction with the restricted conditions. By importing the mathematical models of canard force and moment into the projectile angular motion equations, trim angle induced by canard control force is extracted as the analytical solution of angle of attack increment (AOAI). On this basis, analytical orientations of trajectory angular rate increment and swerving increment are obtained via the frozen coefficient method. A series of simulations under different conditions were implemented to validate the expressions in this effort. Results state that increment orientation of swerving motion can be predicted with available trajectory parameters. The analytical orientations indicate trim value of numerical orientations. Deviations between analytical and numerical orientations relate to initial launch angles and control start time, both lower initial launch angle, and the start time which is closer to the end of flight decreases the deviation convergence time.
\end{abstract}

\section{Introduction}

To maintain flight stability, large caliber spin-stabilized projectiles are launched with high spin rate, usually $150-300 \mathrm{r} / \mathrm{s}$ [1]. High coupling between yaw and pitch flight dynamics makes the cost-effective guidance of spin-stabilized projectile a complex task [2]. To the authors' knowledge, approaches for this problem can be divided into direct methods and indirect methods, respectively.

Dual-spin projectile concept is commonly adopted in the direct methods [3-5]. Projectile swerving dynamics are solved by yaw/pitch channels decoupling controller [6]. The projectile is guided by nominal trajectory tracing [7] or navigation autopilot module [8]. However, yaw/pitch decoupling controller consists of relatively complex mechanisms in the dual-spin concept, and attitude information is necessary in this concept. One of the indirect methods proposed by Fresconi [9] adopts the reduced sensor and actuator requirement concept for spinning projectile's guidance. Projectile spin rate is reduced to about $10 \mathrm{r} / \mathrm{s}$ by installing rear fins; the flight stability is supported by the rear fins. Coupling of pitch and yaw is solved by reducing the gyroscopic effect. However, the rear fin configurations are inapplicable for transforming unguided spin-stabilized projectiles into affordable guided ones. In another indirect method, concept of square crossing fixed-cant canard is adopted $[10,11]$. The subsequent trajectory is predicted by projectile tracking system and steering commands are determined by empirical formula between impact point deviation predicted and orientation of control force. Hence, detailed projectile swerving motion is not taken into consideration. This concept can effectively improve the impact distribution through field test, but the guiding effect is partly affected by the empirical formula unable to vary with practical flight parameters. Further improvement is constrained by this factor. Therefore, guidance strategy involving no empirical formula in direct methods should be considered in the concept.

In the guidance strategy, projectile's target velocity vector angular rate or target swerving orientation will be provided; hence, analytical expression of swerving motion in terms of the available trajectory parameters is necessary. In [12], Burchett et al. proposed a close form solution for dual-spin projectile swerving motion with the pulsejets, which involved initial attitude information. Ollerenshaw and Costello [13] 


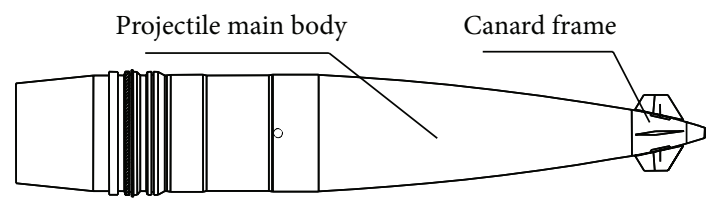

Figure 1: Projectile side view.

deduced a general form expression for the fin- and spinstabilized projectile swerving motion for general force based on the assumption of zero initial Euler angle, while the case does not satisfy this assumption. In this paper, the analytical expressions without attitude information are deduced, which can predict the increment orientation for trajectory angular rate and swerving motion induced by canard control force. This work extends Murphy's theory of gravity-induced trim angle [14] with a nose control force.

\section{Projectile Dynamic Model}

Projectile with the square crossing fixed-cant canard configuration is shown in Figure 1. During the flight, canard frame spins oppositely to projectile main body. Differential canards 1 and 3 provide despun aerodynamic moment to reduce the spin rate of canard frame. The canard frame can be controlled static with respect to the ground at a desired rolling angle by alternator and variable-resistance load system integrated into the nose portion. Attitude sensor is not installed because of large launching overload and projectile high self-spin rate. Available trajectory parameters are velocity vector, position information provided by satellite position receiver, and main body spin rate provided by magnetic sensor. Canard control force is generated from steering canards 2 and 4 when the canard frame despuns at a certain rolling angle. Due to the cant fixed canard, magnitude of control force is uncontrollable and orientation of control force is determined by the rolling angle of canard frame.

2.1. Flight Motions. The mass center and around mass center motions of projectile flight effected by canard control force are descripted by the mathematical model of 6-DOF rigid motions, which are established in four reference frames, respectively, inertial reference frame (IRF: $x_{N^{-}} y_{N}{ }^{-} z_{N}$ ), velocity reference frame (VRF: $x_{2}-y_{2}-z_{2}$ ), no-roll reference frame (NRRF: $\xi-\eta-\zeta)$, and canard reference frame (CRF: $\xi_{c}-\eta_{c}-\zeta_{c}$ ). These reference frames and their relationships are shown in Figures 3 , 4, and 5 .

In the CRF, axle $\xi_{c}$ coincides with projectile body axle $\xi$ in the NRRF, axle $\eta_{c}$ coincides with the direction of canard 1 , and axle $\zeta_{c}$ coincides with the direction of canard 4 in Figure 2 . The axles $\eta_{c}$ and $\zeta_{c}$ rotate around axle $\xi$. Canard rolling-angle $\gamma_{c}$ is defined as the angle between axles $\eta_{c}$ and $\eta$. Canard control force and moment models are established in the CRF.

According to Newton's second law, projectile mass center total velocity vector differential equation is expressed as

$$
m \frac{\mathrm{d} \mathbf{v}}{\mathrm{d} t}=\mathbf{F}
$$

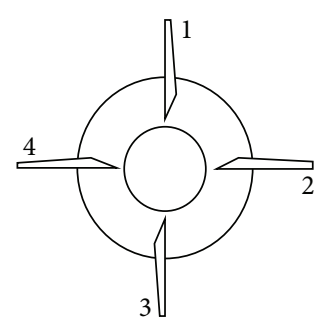

Figure 2: Canard frame front view.

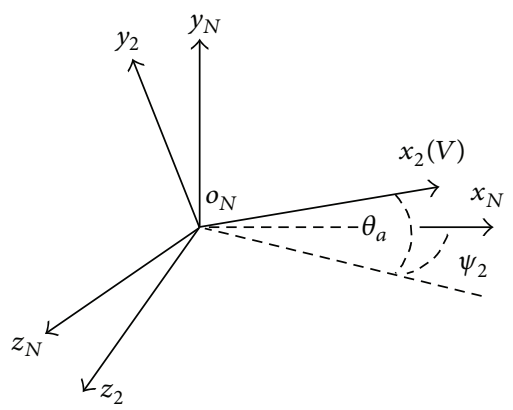

FIGURE 3: Description of the IRF and VRF.

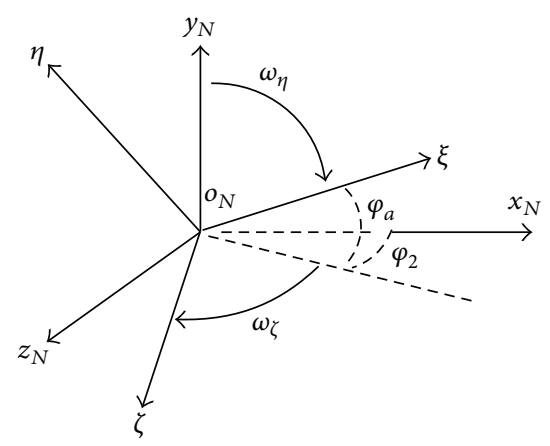

FIgURE 4: Description of the IRF and NRRF.

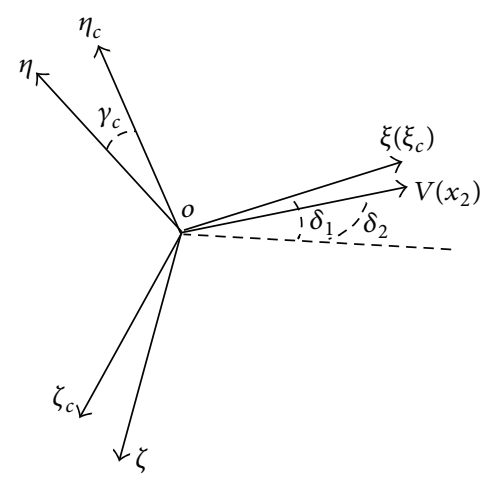

Figure 5: Definition of the NRRF and CRF.

where the total velocity vector $\mathbf{v}$ is composed of velocity vector in the VRF and transport velocity vector induced by rotation of the VRF. The VRF spin-rate vector is expressed as $\boldsymbol{\Omega}_{[2-N]}=\left(\dot{\theta}_{a} \sin \psi_{2},-\dot{\psi}_{2}, \dot{\theta}_{a} \cos \psi_{2}\right)$. Total external force vector $\mathbf{F}$ projectile bearing in the VRF is composed of drag 
vector $\mathbf{R}_{x}$, lift vector $\mathbf{R}_{y}$, Magnus vector $\mathbf{R}_{z}$, gravity vector $\mathbf{G}$, and canard control force vector $\mathbf{F}_{c}$. Equation (1) can be expanded as

$$
m\left(\frac{\partial \mathbf{v}}{\partial t}+\mathbf{\Omega}_{[2-N]} \times \mathbf{v}\right)=\mathbf{R}_{x}+\mathbf{R}_{y}+\mathbf{R}_{z}+\mathbf{G}+\mathbf{F}_{c}
$$

where $\partial \mathbf{v} / \partial t$ means the differential form of velocity vector in the VRF.

Besides, the equations of mass center motion are established in the IRF as

$$
\begin{gathered}
\frac{\mathrm{d} x}{\mathrm{~d} t}=v \cos \psi_{2} \cos \theta_{a}, \quad \frac{\mathrm{d} y}{\mathrm{~d} t}=v \cos \psi_{2} \sin \theta_{a}, \\
\frac{\mathrm{d} z}{\mathrm{~d} t}=v \sin \psi_{2} .
\end{gathered}
$$

According to the theorem of momentum moment, around mass center motion of the projectile can be expressed as

$$
\frac{\mathrm{d} \mathbf{G}}{\mathrm{d} t}=\mathbf{M}
$$

The total momentum moment vector is composed of momentum moment vector $\mathbf{G}$ in the NRRF and transport momentum moment vector induced by rotation of the NRRF. The NRRF spin-rate vector is expressed as $\boldsymbol{\omega}_{1}$. The momentum moment vector is expressed as $\mathbf{G}=\mathbf{J}_{A} \cdot \boldsymbol{\omega}$, where $\mathbf{J}_{A}$ means the inertia matrix and $\boldsymbol{\omega}$ means the Euler angular rate matrix. The total external moment vector projectile bearing in the NRRF is composed of the roll damping moment vector $\mathbf{M}_{x z}$, static moment vector $\mathbf{M}_{z}$, pitch damping moment vector $\mathbf{M}_{z z}$, Magnus moment vector $\mathbf{M}_{\mathbf{y}}$, and canard inducing control moment vector $\mathbf{M}_{c}$. Equation (4) can be expanded as

$$
\frac{\partial \mathbf{G}}{\partial t}+\omega_{1} \times \mathbf{G}=\mathbf{M}_{z}+\mathbf{M}_{y}+\mathbf{M}_{x z}+\mathbf{M}_{z z}+\mathbf{M}_{c}
$$

where $\partial \mathrm{G} / \partial t$ means the differential form of the momentum moment vector in the NRRF. The around mass center angular motion differential equations can be obtained by integrating expanded form of (5). For brevity, detailed expressions of force and moment are listed in the appendix.

2.2. Canard Force and Moment. Canard control force vector is considered sum of aerodynamic force induced by the four wings. Force vector provided by differential canards 1 and 3 in the CRF is indicated as $\mathbf{F}_{c d}$ with orientation vertical to rolling angle and magnitude $F_{c d}$; force vector provided by steering canards 2 and 4 in the CRF is indicated as $\mathbf{F}_{p d}$ with orientation parallel to rolling angle and magnitude $F_{p d}$.
Expression of canard force vector $\mathbf{F}_{c}$ in the VRF can be obtained by reference frame transforming:

$$
\begin{aligned}
\mathbf{F}_{c}= & \boldsymbol{\Omega}_{[\xi-2]} \cdot \boldsymbol{\Omega}_{[\xi \mathcal{\xi}-\xi]} \cdot\left(\mathbf{F}_{c d}+\mathbf{F}_{p d}\right) \\
= & {\left[\begin{array}{ccc}
\cos \delta_{2} \cos \delta_{1} & \cos \delta_{2} \cos \delta_{1} & \sin \delta_{2} \\
-\sin \delta_{1} & \cos \delta_{1} & 0 \\
-\sin \delta_{2} \cos \delta_{1} & -\sin \delta_{2} \sin \delta_{1} & \cos \delta_{2}
\end{array}\right] } \\
& \cdot\left(\left[\begin{array}{c}
0 \\
-\sin \gamma_{c} F_{c d} \\
\cos \gamma_{c} F_{c d}
\end{array}\right]+\left[\begin{array}{c}
0 \\
\cos \gamma_{c} F_{p d} \\
\sin \gamma_{c} F_{p d}
\end{array}\right]\right),
\end{aligned}
$$

where $\Omega_{[\xi-2]}$ means transforming matrix from the NRRF to VRF and $\Omega_{\left[\xi_{\mathcal{c}-\xi}\right]}$ means transforming vector from the CRF to NRRF.

Canard control moment vector $\mathbf{M}_{c}$ in the NRRF is considered sum of moment vector $\mathbf{M}_{c d}$ by differential canards 1 and 3 and $\mathbf{M}_{p d}$ by steering canards 2 and 4 as

$$
\mathbf{M}_{c}=\mathbf{M}_{c d}+\mathbf{M}_{p d}=\left[\begin{array}{c}
0 \\
\sin \gamma_{c} M_{c d} \\
\cos \gamma_{c} M_{c d}
\end{array}\right]+\left[\begin{array}{c}
0 \\
\cos \gamma_{c} M_{p d} \\
-\sin \gamma_{c} M_{p d}
\end{array}\right] .
$$

According to the relationship between force and moment $M_{c d}=F_{c d} \times X_{0}$ and $M_{p d}=F_{p d} \times X_{0}$, where $X_{0}$ means the axial distance between mass center and pressure center of canard frame.

In this section, models of projectile flight motion and canard control force and moment for numerical solution of swerving motion are established. Note that some small magnitude aerodynamic forces are ignored, such as drag force and roll damping moment brought to the projectile main body by the canard frame. Moreover, aerodynamic force yielded by canard 1 shows similar magnitude and opposite direction with force yielded by canard 3, hence the balance force and moment of them $F_{c d}, M_{c d}$ can be neglected to simplify the deduce process of analytical swerving motion expressions.

\section{Angular Motion Linearization}

In the 6-DOF mathematical model, angular motions in mass center and around mass center motions are highly coupled. To linearize the angular motion, 6-DOF model is processed with the following assumptions:

(a) ignore the products of small magnitude values and high order of them;

(b) ignore the asymmetric of the projectile geometric parameters and eccentricity of mass center;

(c) consider the AOA small magnitude values, and assume the following approximation:

$$
\begin{gathered}
\sin \delta_{1} \approx \delta_{1}, \quad \sin \delta_{2} \approx \delta_{2}, \\
\cos \delta_{1} \approx 1, \quad \cos \delta_{2} \approx 1, \\
\beta \approx 0, \quad \delta_{1} \approx \phi_{a}-\theta_{a}, \quad \delta_{2} \approx \phi_{2}-\psi_{2} ;
\end{gathered}
$$


(d) ignore the drag force $F_{x c}$ and roll damping moment $M_{\xi c}$ induced by fixed-cant canard frame;

(e) ignore the $F_{c d}$ and $M_{c d}$ yielded by differential canards 1 and 3; assume the magnitude approximation: $F_{c} \approx$ $F_{p d}, M_{c} \approx F_{p d} \times X_{0}$.

Then, (2) and (5) are expanded and simplified by the above rules. Differential equations of trajectory path and deflection angle and second-order differential equations of pitch and yaw angle are obtained as follows:

$$
\begin{aligned}
\dot{\theta}_{a}= & \frac{\left(Q v^{2} c_{y}^{\prime} \delta_{1}+Q v^{2} c_{z}^{\prime} \delta_{2}-m g \cos \theta_{a}+\cos \gamma_{c} F_{c}\right)}{m v}, \\
\dot{\psi}_{2}= & \frac{\left(Q v^{2} c_{y}^{\prime} \delta_{2}-Q v^{2} c_{z}^{\prime} \delta_{1}+m g \sin \theta_{a} \sin \psi_{2}+\sin \gamma_{c} F_{c}\right)}{m v}, \\
\ddot{\varphi}_{a}= & \left(-Q l v^{2} m_{z}^{\prime} \delta_{1}-Q l d v \omega_{\zeta} m_{z z}^{\prime}-Q l d \omega_{\xi} v m_{y}^{\prime \prime} \delta_{2}\right. \\
& \left.+C \omega_{\xi} \omega_{\eta}+\omega_{\eta} \omega_{\zeta} \tan \phi_{2}+X_{0} \cos \gamma_{c} F_{c}\right)(A)^{-1} \\
\ddot{\varphi}_{2}= & \left(Q l v^{2} m_{z}^{\prime} \delta_{2}+Q l d v \omega_{\eta} m_{z z}^{\prime}-Q l d \omega_{\xi} v m_{y}^{\prime \prime} \delta_{1}\right. \\
& \left.+C \omega_{\xi} \omega_{\zeta}-\omega_{\eta}{ }^{2} \tan \phi_{2}+X_{0} \sin \gamma_{c} F_{c}\right)(A)^{-1} .
\end{aligned}
$$

The complex trajectory angle differential equation is obtained by adding (9) with (10) multiplied by imaginary unit $i$ as

$$
\dot{\Psi}=\left(b_{y} v-i b_{z} \dot{\gamma}\right) \Delta+\frac{F_{c}}{m v} e^{i \gamma_{c}},
$$

where $b_{y}=Q v c_{y}^{\prime} / m, b_{z}=Q v c_{z}^{\prime} / m, e^{i \gamma_{c}}=\cos \gamma_{c}+i \sin \gamma_{c}$, $\dot{\Psi}=\theta_{a}+i \psi_{2}, \dot{\Delta}=\delta_{1}+i \delta_{2}$. Differentiating (13) with respect to time yields

$$
\ddot{\Psi}=\left(b_{y} \dot{v}-i b_{z} \ddot{\gamma}\right) \Delta+\left(b_{y} v-i b_{z} \dot{\gamma}\right) \dot{\Delta}-\frac{\dot{v} F_{c}}{m v^{2}} e^{i \gamma_{c}} .
$$

Meanwhile, complex Euler angle differential equation is obtained by adding (11) with (12) multiplied by $-i$ as

$$
\ddot{\boldsymbol{\Phi}}+\left(k_{z z} v-i \frac{C}{A} \dot{\gamma}\right) \dot{\boldsymbol{\Phi}}=\left(k_{z} v^{2}+k_{y} v\right) \Delta+\frac{X_{0}}{A} F_{c} e^{i \gamma_{c}},
$$

where $k_{z}=Q l m_{z}^{\prime} / A, k_{z z}=Q l^{2} m_{z z}^{\prime} / A, k_{y}=Q l d k_{y}^{\prime \prime} / A, \dot{\Phi}=$ $\varphi_{a}+i \varphi_{2}$.

Consider the relationships between trajectory angle, Euler angle, and angle of attack: $\ddot{\Phi}=\ddot{\Psi}+\ddot{\Delta}, \dot{\Phi}=\dot{\Psi}+\dot{\Delta}$. Complex angle of attack (AOA) motion can be obtained by importing (13) and (14) into (15). The expression of complex AOA differential equation is shown as in formula (17).

In order to linearize the complex AOA differential equation, time infinitesimal $\mathrm{d}(t)$ is substituted by dimensionless trajectory arc infinitesimal $\mathrm{d}(s)$, using the following derivation form:

$$
\begin{gathered}
\dot{v}=v \cdot v^{\prime} ; \quad \dot{\Delta}=v \cdot \Delta^{\prime} ; \\
\ddot{\Delta}=v^{2} \cdot \Delta^{\prime \prime}-v^{2} \cdot \Delta^{\prime}\left(b_{x}+\frac{g \sin \theta}{v^{2}}\right) .
\end{gathered}
$$

The factors $v^{2}$ and $v$ in (17) are eliminated by importing the above relationships, and (17) can be linearized as (18):

$$
\begin{aligned}
\ddot{\Delta}+ & \left(k_{z z}+b_{y}-i \frac{C \dot{\gamma}}{A v}\right) v \dot{\Delta}-\left[k_{z}+i \frac{C \dot{\gamma}}{A v}\left(b_{y}-\frac{A}{C} k_{y}\right)\right] v^{2} \Delta \\
= & -\ddot{\theta}-\dot{\theta} v\left(k_{z z}-i \frac{C \dot{\gamma}}{A v}\right) \\
& +\left(\frac{X_{0}}{A}+\frac{\dot{v}}{m v^{2}}-\frac{k_{z z}}{m}+i \frac{C \dot{\gamma}}{A m v}\right) F_{c} e^{i \gamma_{c}} \\
\Delta^{\prime \prime}+ & (H-i P) \Delta^{\prime}-(M+i P T) \Delta \\
= & -\frac{\ddot{\theta}}{v^{2}}-\frac{\dot{\theta}}{v}\left(k_{z z}-i P\right) \\
& +\left(\frac{X_{0}}{A}+\frac{\dot{v}}{m v^{2}}-\frac{k_{z z}}{m}+i \frac{C \dot{\gamma}}{A m v}\right) \frac{F_{c}}{v^{2}} e^{i \gamma_{c}}
\end{aligned}
$$

where $H=k_{z z}+b_{y}-b_{x}-g \sin \theta / v^{2}, P=C \dot{\gamma} / A v, M=k_{z}$, and $T=b_{y}-k_{y} A / C$.

\section{Analytical Solutions}

4.1. Complex AOAI Solution. To deduce the solution of linear nonhomogeneous differential equation (18), first, we assume the general solution form of homogeneous equation as

$$
\Delta=C_{1} e^{l_{1} s}+C_{2} e^{l_{2} s}
$$

where $C_{1}$ and $C_{2}$ are indeterminate constants and $l_{1}$ and $l_{2}$ are eigenvalues of homogeneous equation; hence, the following equations are set up:

$$
\begin{gathered}
l_{1,2}^{2}+(H-i P) l_{1,2}-(M+i P T)=0, \\
l_{1}+l_{2}=-(H-i P) \\
l_{1} \cdot l_{2}=-(M+i P T) .
\end{gathered}
$$

Differentiating (19) with respect to trajectory arc yields

$$
\Delta^{\prime}=C_{1}^{\prime}(s) e^{l_{1} s}+C_{2}^{\prime}(s) e^{l_{2} s}+C_{1} l_{1}^{2} e^{l_{1} s}+C_{2} l_{2}^{2} e^{l_{2} s} .
$$

Assuming $C_{1}^{\prime} e^{l_{1} s}+C_{2}^{\prime} e^{l_{2} s}=0$ and differentiating (21) with respect to trajectory arc yield

$$
\Delta^{\prime \prime}=C_{1}^{\prime}(s) l_{1} e^{l_{1} s}+C_{2}^{\prime}(s) l_{2} e^{l_{2} s}+C_{1} l_{1}^{2} e^{l_{1} s}+C_{2} l_{2}^{2} e^{l_{2} s} .
$$

Importing (19), (20a), (20b), (20c), (21), and (22) into (18) yields

$$
\begin{aligned}
C_{1}^{\prime} l_{1} e^{l_{1} s}+C_{2}^{\prime} l_{2} e^{l_{2} s} \\
=-\frac{\ddot{\theta}}{v^{2}}-\frac{\dot{\theta}}{v}\left(k_{z z}-i P\right) \\
\quad+\left(\frac{X_{0}}{A}+\frac{\dot{v}}{m v^{2}}-\frac{k_{z z}}{m}+i \frac{C \dot{\gamma}}{A m v}\right) \frac{F_{c}}{v^{2}} e^{i \gamma_{c}}
\end{aligned}
$$


Solution of simultaneous expressions equation (23) and $C_{1}^{\prime} e^{l_{1} s}+C_{2}^{\prime} e^{l_{2} s}=0$ is written as

$$
\begin{aligned}
& C_{1,2}^{\prime}(s) \\
& \begin{aligned}
= \pm \frac{1}{l_{1}-l_{2}}[ & -\frac{\ddot{\theta}}{v^{2}}-\frac{\dot{\theta}}{v}\left(k_{z z}-i P\right) \\
& \left.+\left(\frac{X_{0}}{A}+\frac{\dot{v}}{m v^{2}}-\frac{k_{z z}}{m}+i \frac{C \dot{\gamma}}{A m v}\right) \frac{F_{c}}{v^{2}} e^{i \gamma_{c}}\right] e^{-l_{1,2} s}
\end{aligned}
\end{aligned}
$$

Then, constants $C_{1}, C_{2}$ are determined by integrating (24). Considering $\ddot{\theta}, \dot{\theta}$ variables, the integrating result is expressed as

$$
\begin{aligned}
& C_{1,2}(s) \\
& = \pm \frac{1}{l_{1}-l_{2}}\left[\frac{\ddot{\theta}}{v^{2} l_{1,2}}+\frac{\ddot{\theta}}{v^{2} l_{1,2}^{2}}\left(k_{z z}-i P\right)+\frac{\dot{\theta}}{v l_{1,2}}\right. \\
& \left.\quad \times\left(k_{z z}-i P\right)\right] e^{-l_{1,2} s} \\
& \mp\left(\frac{m X_{0}}{A}+\frac{\dot{v}}{v^{2}}-k_{z z}+i \frac{C \dot{\gamma}}{A v}\right) \\
& \times \frac{F_{c}}{m v^{2} l_{1,2}} e^{-l_{1,2} s+i \gamma_{c}} .
\end{aligned}
$$

Finally, importing (20b), (20c), and (25) into (19) yields the particular solution of the complex angular motion as

$$
\begin{aligned}
\Delta= & \frac{1}{M+i P T}\left[\frac{\ddot{\theta}}{v^{2}}+\frac{\dot{\theta}}{v}\left(k_{z z}-i P\right)\right]+\frac{k_{z z}-i P}{v^{2}} \times \frac{H-i P}{M+i P T} \ddot{\theta} \\
& -\frac{1}{M+i P T}\left(\frac{m X_{0}}{A}+\frac{\dot{v}}{v^{2}}-k_{z z}+i \frac{C \dot{\gamma}}{A v}\right) \frac{F_{c}}{m v^{2}} e^{i \gamma_{c}} .
\end{aligned}
$$

The trim angle inducing by the canard control force is indicated by the items containing parameter $F_{c}$. By observing right-hand items of particular solution, the analytical expression of complex AOAI induced by canard control force can be extracted as

$$
\begin{aligned}
\Delta_{c} & =\delta_{1 c}+i \delta_{2 c} \\
& =-\frac{1}{M+i P T}\left(\frac{m X_{0}}{A}+\frac{\dot{v}}{v^{2}}-k_{z z}+i \frac{C \dot{\gamma}}{A v}\right) \frac{F_{c}}{m v^{2}} e^{i \gamma_{c}} .
\end{aligned}
$$

Transforming the denominator into real numbers and omitting small magnitude values, (27) is expressed in a short form as

$$
\begin{aligned}
& \delta_{1 c}=\left[K_{1} \cos \gamma_{c}-K_{2} \sin \gamma_{c}\right] \frac{F_{c}}{m v^{2}}, \\
& \delta_{2 c}=\left[K_{2} \cos \gamma_{c}+K_{1} \sin \gamma_{c}\right] \frac{F_{c}}{m v^{2}},
\end{aligned}
$$

where

$$
\begin{aligned}
K_{1} & =-\frac{m X_{0}}{M A}-\frac{\dot{v}}{M v^{2}}-\frac{P^{2} T}{M^{2}}, \\
K_{2} & =\frac{m X_{0} P T}{M^{2} A}+\frac{\dot{v} P T}{M^{2} v^{2}}+\frac{P}{M} .
\end{aligned}
$$

4.2. Complex TARI Solution. The frozen coefficient method is adopted to deduce analytical solutions of trajectory parameter increment in swerving motion. The angular rate of velocity vector in VRF is expressed as trajectory path angular rate in plane $o-x_{2}-y_{2}$ and deflection angular rate in plane $o-x_{2}-$ $z_{2}$. The trajectory angular rate increment (TARI) indicates the orientation of velocity vector rotation. The differential equations of trajectory angular rate with canard control force refer to (9) and (10); trajectory angular rate increment (TARI) for canard control force is obtained by subtracting the differential equation of trajectory angular rate without canard control force. For brevity, result is written as

$$
\left[\begin{array}{l}
\dot{\theta}_{a c} \\
\dot{\psi}_{2 c}
\end{array}\right]=\left[\begin{array}{c}
\delta_{1 c} \\
\delta_{2 c}
\end{array}\right] b_{y} v+\left[\begin{array}{c}
\delta_{2 c} \\
-\delta_{1 c}
\end{array}\right] b_{z} v+\left[\begin{array}{c}
\cos \gamma_{c} \\
\sin \gamma_{c}
\end{array}\right] \cdot \frac{F_{c}}{m v} .
$$

According to the main principle of frozen coefficient method, slowly varying trajectory parameters are considered as constants in a short time, such as the total velocity, dynamic pressure, and aerodynamic coefficients. Submitting complex AOAI analytical solutions $\delta_{1 c}, \delta_{2 c}$ to (30) yields

$$
\begin{gathered}
{\left[\begin{array}{c}
\dot{\theta}_{a c} \\
\dot{\psi}_{2 c}
\end{array}\right]=\left[\begin{array}{c}
M_{1} \cos \gamma_{c}+M_{2} \sin \gamma_{c} \\
-M_{2} \cos \gamma_{c}+M_{1} \sin \gamma_{c}
\end{array}\right] \cdot \frac{F_{c}}{m v},} \\
M_{1}=b_{y} K_{1}+b_{z} K_{2}+1 \\
M_{2}=b_{z} K_{1}-b_{y} K_{2} .
\end{gathered}
$$

4.3. Increment Orientations. Differential equation of mass center swerving motion with canard control force referring to (4) is written as

$$
\begin{gathered}
\dot{y}=\cos \left(\psi_{2}+\Delta t \cdot \dot{\psi}_{2 c}\right) \sin \left(\theta_{a}+\Delta t \cdot \dot{\theta}_{a c}\right) \cdot v, \\
\dot{z}=\sin \left(\psi_{2}+\Delta t \cdot \dot{\psi}_{2 c}\right) \cdot v .
\end{gathered}
$$

Assuming that the short time is $\Delta t, \sin \left(\dot{\theta}_{a} \cdot \Delta t\right) \approx \dot{\theta}_{a} \cdot \Delta t$, $\sin \left(\dot{\psi}_{2} \cdot \Delta t\right) \approx \dot{\psi}_{2} \cdot \Delta t, \cos \left(\dot{\theta}_{a} \cdot \Delta t\right) \approx 1$, and $\cos \left(\dot{\psi}_{2} \cdot \Delta t\right) \approx 1$.

Subtracting (32a) and (32b) with differential equation of mass center swerving motion without canard control force, projectile swerving increment induced by the canard control force in short time $\Delta t$ is obtained as

$$
\begin{gathered}
y_{c}=\left(\cos \psi_{2} \cos \theta_{a} \cdot \dot{\theta}_{a c}-\sin \psi_{2} \sin \theta_{a} \cdot \dot{\psi}_{2 c}\right. \\
\left.-\cos \theta_{a} \sin \psi_{2} \cdot \dot{\theta}_{a c} \cdot \dot{\psi}_{2 c} \cdot \Delta t\right) \cdot v \cdot \Delta t^{2}, \\
z_{c}=\cos \psi_{2} \cdot \dot{\psi}_{2 c} \cdot v \cdot \Delta t^{2} .
\end{gathered}
$$



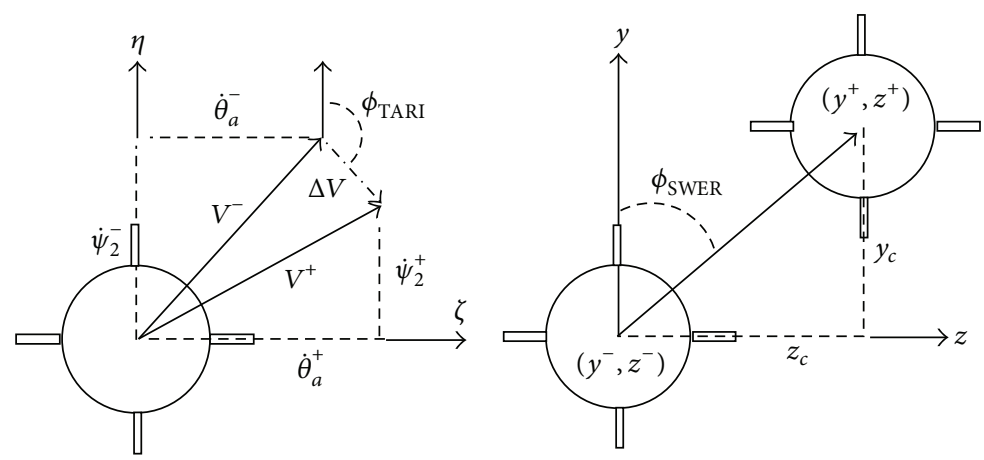

FIGURE 6: Rear view of the projectile, definition of $\phi_{\text {TARI }}, \phi_{\text {SWER }}$ "-" means uncontrolled state, “+” means controlled state. The positive $\xi$ and $x$ point into the page.

TABLE 1: Parameters involved in analytical expressions.

\begin{tabular}{lccc}
\hline $\begin{array}{l}\text { Constant } \\
\text { coefficient }\end{array}$ & $\begin{array}{c}\text { Variable } \\
\text { coefficient }\end{array}$ & $\begin{array}{c}\text { Trajectory } \\
\text { parameters }\end{array}$ & Control input \\
\hline$m$ & $X_{0}$ & $v$ & \\
$A$ & $k_{z}$ & $\theta_{a}$ & \\
$C$ & $b_{x}$ & $\psi_{2}$ & $F_{c}$ \\
$g$ & $b_{y}$ & $\dot{\gamma}$ & \\
$\Delta t$ & $k_{y}$ & $\dot{v}$ & \\
\hline
\end{tabular}

Simple algebraic manipulation yields the analytical expressions of orientations of TARI and swerving increment, shown in Figure 6:

$$
\begin{gathered}
\phi_{\mathrm{TARI}}=\tan ^{-1}\left(\frac{\dot{\psi}_{2 c}}{\dot{\theta}_{a c}}\right), \quad \dot{\psi}_{2 c} \leq 0, \\
\phi_{\mathrm{TARI}}=\tan ^{-1}\left(\frac{\dot{\psi}_{2 c}}{\dot{\theta}_{a c}}\right)+180, \quad \dot{\psi}_{2 c}>0, \\
\phi_{\mathrm{SWER}}=\tan ^{-1}\left(\frac{z_{c}}{y_{c}}\right), \quad z_{c} \leq 0, \\
\phi_{\mathrm{SWER}}=\tan ^{-1}\left(\frac{z_{c}}{y_{c}}\right)+180, \quad z_{c}>0 .
\end{gathered}
$$

All parameters involved in analytical expressions are listed in Table 1. Real-time performance of analytical solutions is ensured by the variable coefficients, which are updated with the trajectory parameters and aerodynamic coefficients during the flight. Combining with the trajectory parameters and constant coefficients, the orientations of TARI and swerving increment with canard control input can be predicted. Note that the flying stability of the projectile should exist.

\section{Simulation Results}

In this chapter, numerical simulations of projectile 6-DOF rigid motions with and without canard control were implemented. Numerical solutions of trajectory parameter increments are obtained by subtraction operation. Meanwhile,
TABLE 2: Projectile physical properties.

\begin{tabular}{lccccc}
\hline$m(\mathrm{~kg})$ & $X_{c}(\mathrm{~m})$ & $C$ & $A$ & $L(\mathrm{~m})$ & $D(\mathrm{~m})$ \\
\hline 45 & 0.343 & 0.16 & 1.8 & 0.9 & 0.155 \\
\hline
\end{tabular}

TABLE 3: Initial launch conditions.

\begin{tabular}{lccc}
\hline \multicolumn{2}{c}{ Launch conditions } & \multicolumn{2}{c}{ Meteorological conditions } \\
\hline Velocity & $930 \mathrm{~m} / \mathrm{s}$ & Ground pressure & $1000 \mathrm{hPa}$ \\
Elevation & $50 \mathrm{deg}$ & Virtual temperature & 288.9 \\
Direction & $0 \mathrm{deg}$ & Longitudinal wind & $0 \mathrm{~m} / \mathrm{s}$ \\
Spin rate & $1800 \mathrm{rad} / \mathrm{s}$ & Lateral wind & $0 \mathrm{~m} / \mathrm{s}$ \\
\hline
\end{tabular}

analytical solutions of TARI and swerving increment are calculated by equations in the above section, and orientation deviations are analyzed by comparing with the numerical solutions.

5.1. Simulation Configurations. Projectile physical properties referring to the actual parameters are listed in Table 2, and the initial launch conditions are listed in Table 3. The fourthorder Runge-Kutta method is employed in the numerical simulations, and the step-size is $0.00001 \mathrm{~s}$.

Projectile aerodynamic coefficients are obtained by a series of computational fluid dynamics simulations previously. All coefficients are expressed as function of Mach number, shown in Figure 7. The numerical unguided 6-DOF rigid trajectory result is in good agreement with the firing table data of the conventional $155 \mathrm{~mm}$ spin-stabilized projectile.

5.2. Numerical Trajectory Results. Trajectory simulations of $155 \mathrm{~mm}$ spin-stabilized projectile with and without canard control force are implemented under the same initial conditions. Control period is set as the duration from apogee point (50 sec) to impact point, and the canard rolling-angle $\gamma_{c}$ is set as $0 \mathrm{deg}$.

Figure 8 shows the results of trajectory numerical simulations. Impact point drift of controlled trajectory indicates $(\Delta x, \Delta z)=(132.0 \mathrm{~m}, 75.3 \mathrm{~m})$ and the impact point $\mathrm{drift}$ 


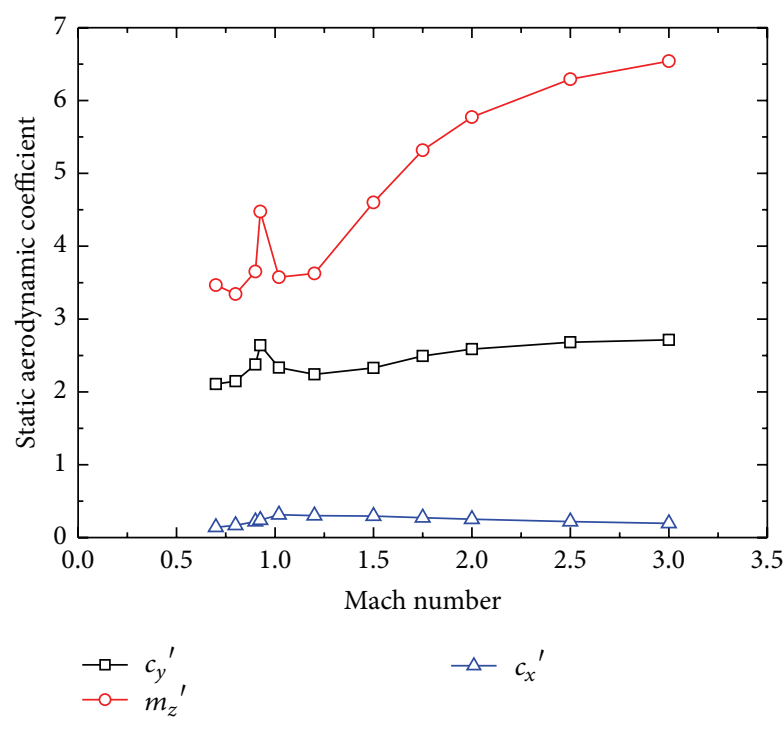

(a) Static aerodynamic coefficients versus Mach number

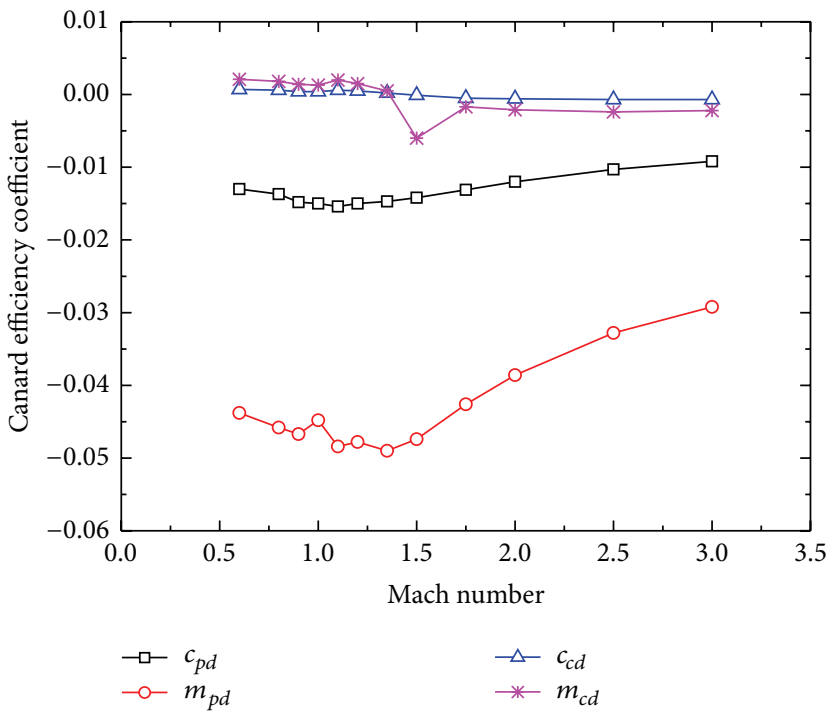

(c) Canard efficiency coefficients versus Mach number

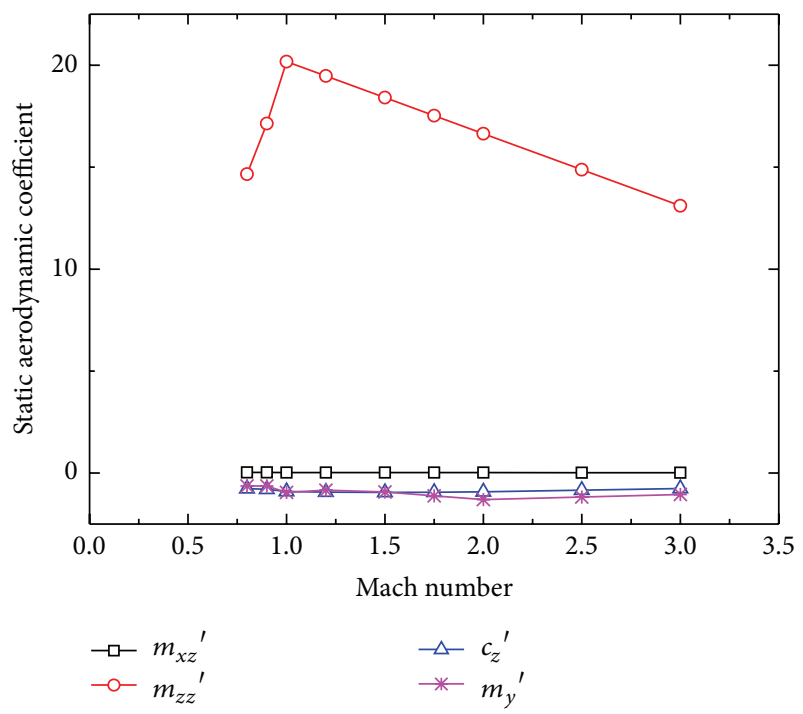

(b) Dynamic aerodynamic coefficients versus Mach number

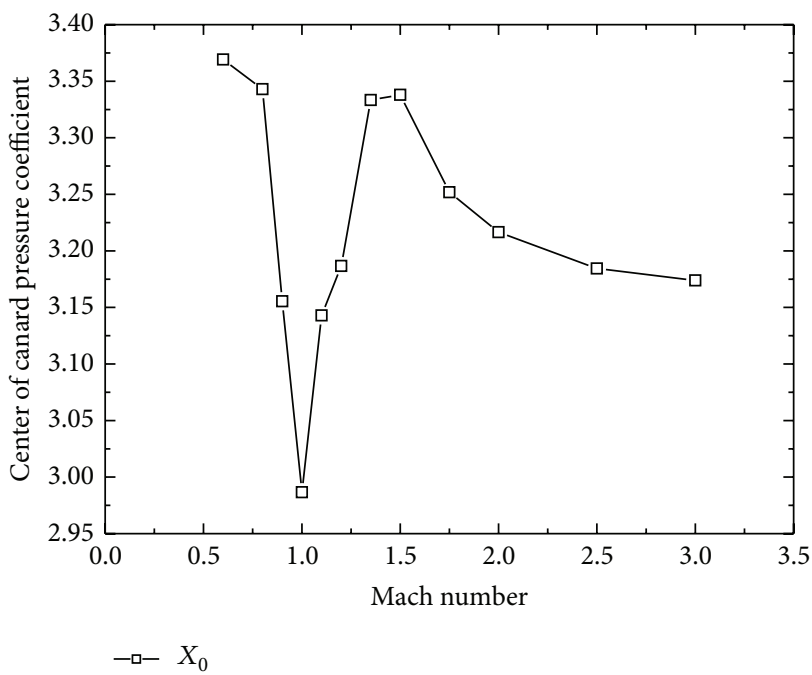

(d) Canard pressure center coefficient versus Mach number

FIgURE 7: Aerodynamic coefficients adopted in 6-DOF mathematical model.

orientation indicates $29.7 \mathrm{deg}$. Inconsistency can be seen from the impact point drift orientation and canard rolling angle.

One of the influence factors is the nonconcentrated control force orientation caused by the gyroscopic effect, which can be reflected by the angular motion of the projectile. However, the angular motion around the center of mass is indicated by the AOAI, and the angular motion of velocity vector is indicated by the TARI. To observe these angular motions, 3D curves of complex AOAI and TARI with time history are shown in Figures 9 and 10.

Damped oscillation waves can be seen in numerical solutions of complex AOAI and TARI with similar frequency. According to the control system stability criterion, the angular motions converge to steady state at about $20 \mathrm{sec}$ after canard control started. Therefore, the adoptive prediction orientations should be steady state value or trim value of the oscillation process.

5.3. Analytical Angular Motion Results. Combining with the known meteorological parameters, aerodynamic coefficient and rolling-angle $\gamma_{c}$, analytical solution of the AOAI is calculated via (28a) and (28b) by using the controlled trajectory parameters. Moreover, analytical solution of TARI is calculated by (31a), (31b), and (31c). For the convenience of orientation observation, projection of analytical and numerical complex AOAI and TARI in plane of $o-\eta-\zeta$ and $o-y_{2}-z_{2}$ is shown in Figures 11 and 12. Due to the approximate process for the deduction of analytical expression, magnitude deviation can be seen from the comparison. However, our attention is concentrated in the orientation prediction. By comparing 


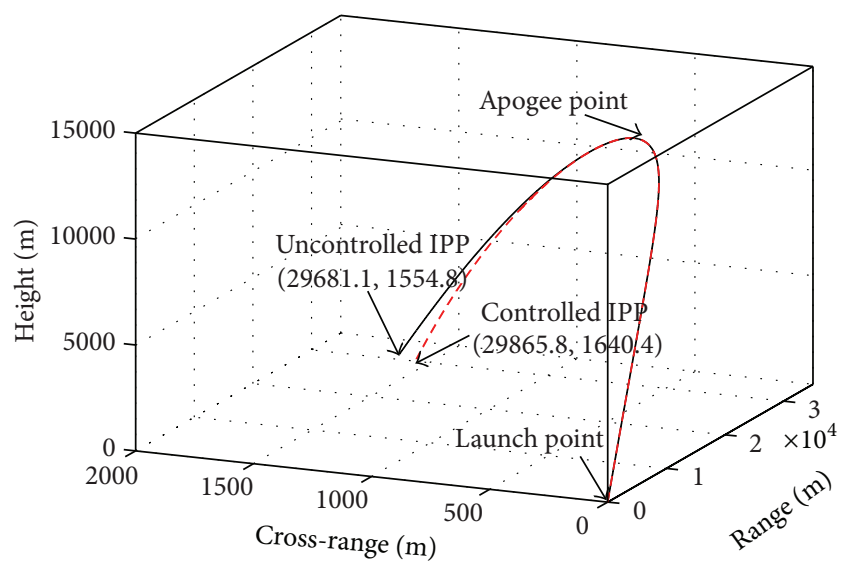

- - Controlled trajectory

_ Uncontrolled trajectory

FIGURE 8: Projectile 3D trajectory comparison.

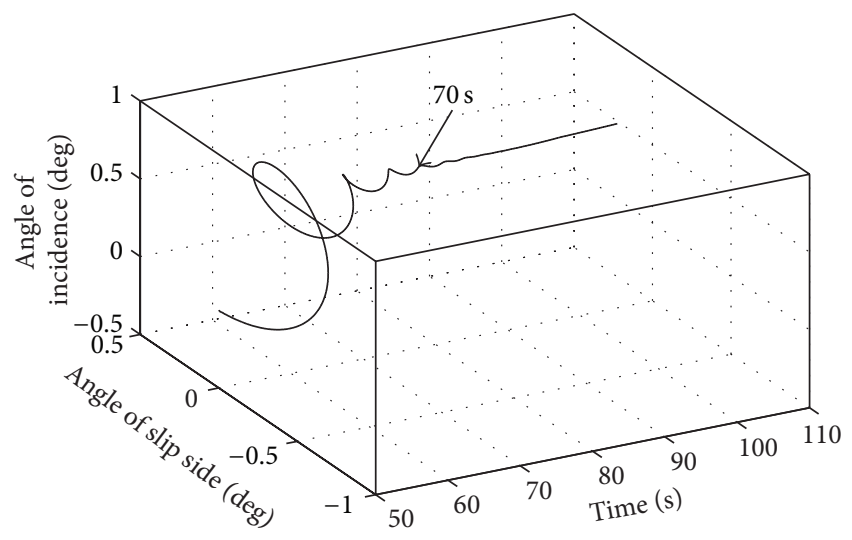

FIgURE 9: 3D curve of complex AOAI with time history.

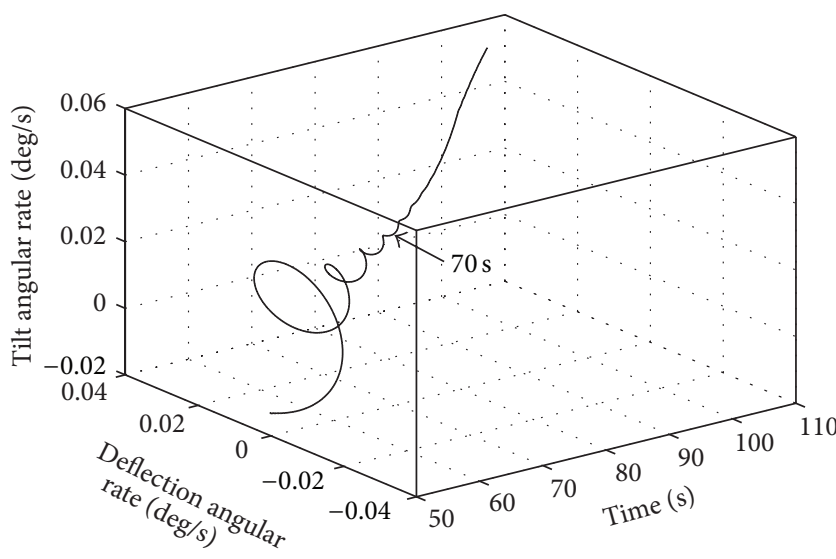

FIGURE 10: 3D curve of complex TARI with time history.

the orientations of analytical solutions with numerical steady state value, well agreement can be seen in this case, and analytical orientations indicate the trim value of numerical orientations. To discuss general applicability of analytical orientation expressions for TARI and swerving increment, more simulations are implemented in the following section.

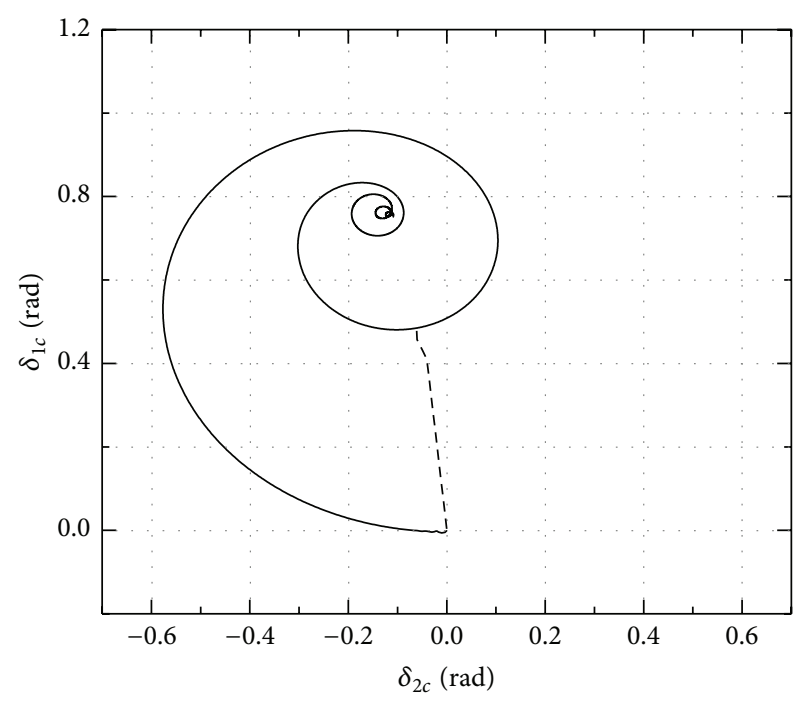

_ Numerical solution

- - Analytical solution

FIGURE 11: Projection of complex AOAI in plane of $o-\eta-\zeta$.

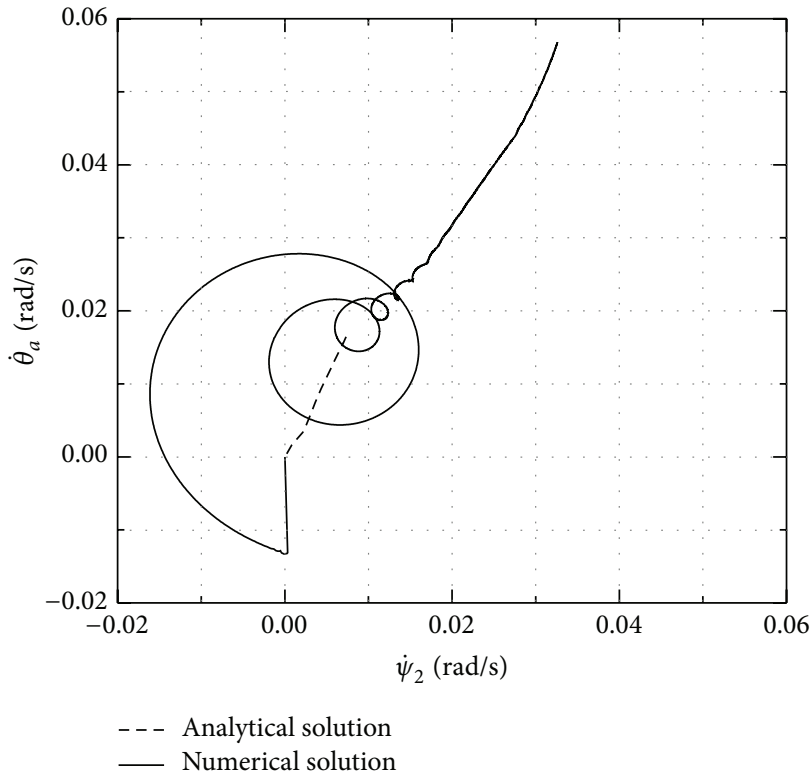

FIgURE 12: Projection of complex TARI in plane of $o-y_{2}-z_{2}$.

5.4. Orientation Prediction. Under different conditions, analytical and numerical orientations of TARI and swerving increment were calculated. The start time of control period is set at the apogee point, $15 \mathrm{~s}$ later, and $30 \mathrm{~s}$ later. The initial elevations are set as 50 deg., 40 deg., and $30 \mathrm{deg}$. The numerical and analytical orientations of TARI and swerving increment are drawn in Figures 13, 14, and 15.

As seen from the left side figures, analytical TARI orientations are not affected by initial elevation and control start time and appear as trim value of numerical orientations. Consider that the deviations between numerical and analytical orientations achieve steady state when the amplitude of oscillation is 


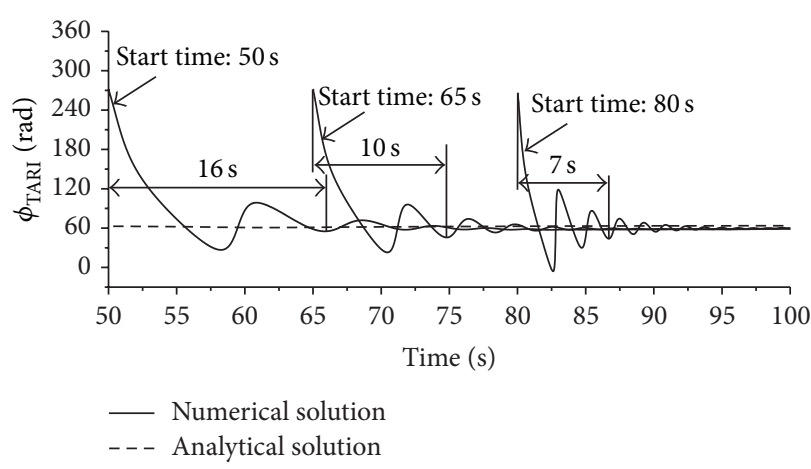

(a) TARI orientation

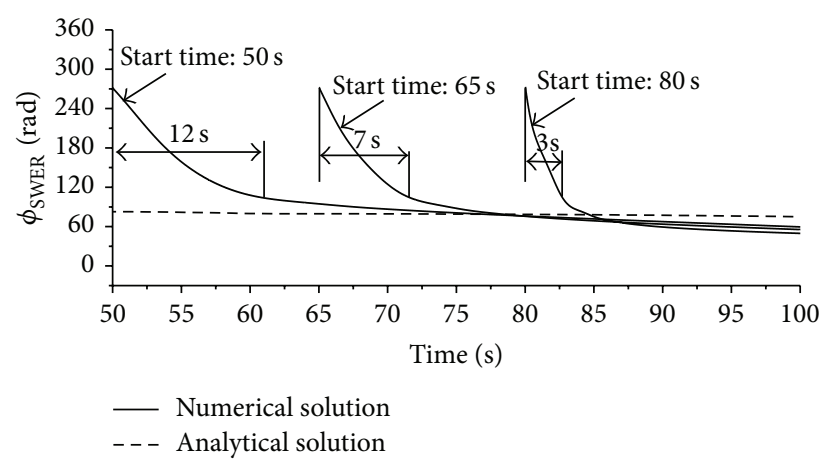

(b) Swerving increment orientation

FIGURE 13: Numerical and analytical results under initial elevation $50 \mathrm{deg}$.

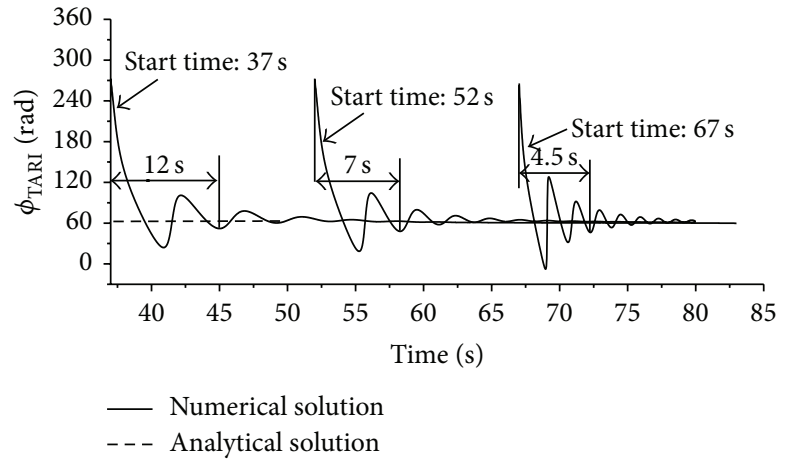

(a) TARI orientation

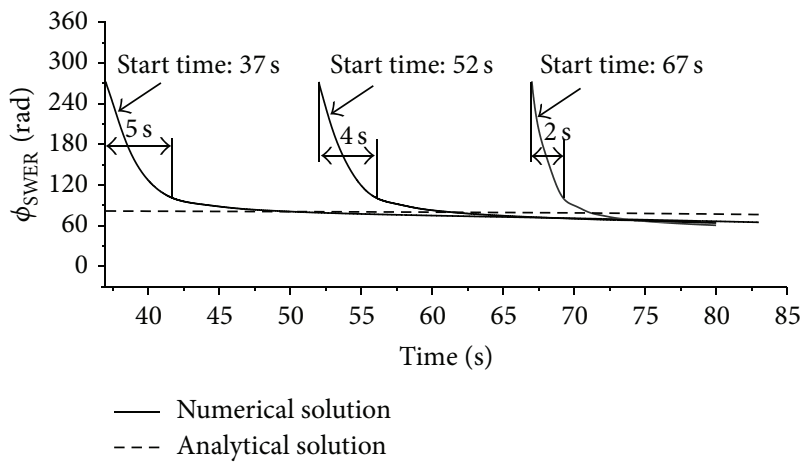

(b) Swerving increment orientation

FIGURE 14: Numerical and analytical results under initial elevation $40 \mathrm{deg}$.

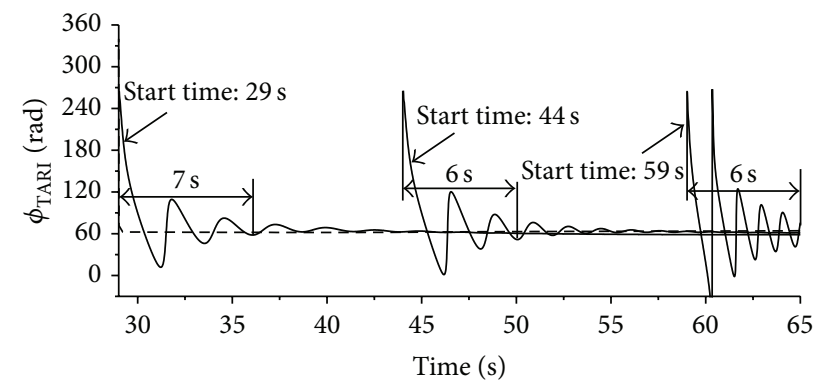

- Numerical solution - - Analytical solution

(a) TARI orientation

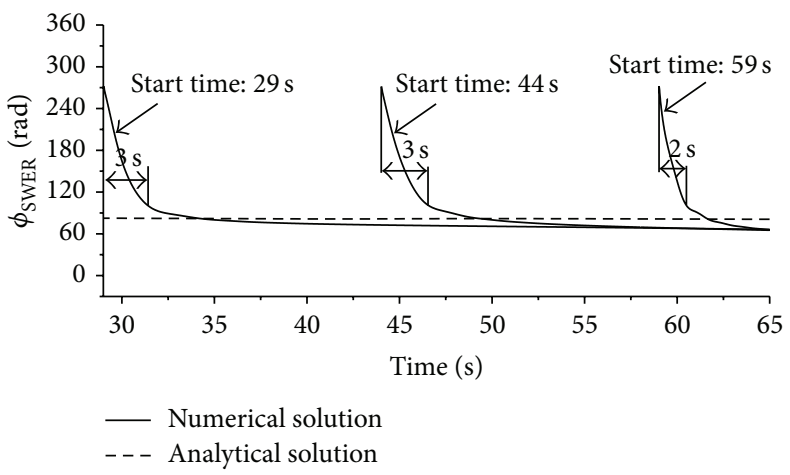

(b) Swerving increment orientation

FIGURE 15: Numerical and analytical results under initial elevation $30 \mathrm{deg}$.

less than $10 \mathrm{deg}$. Convergence time for the deviation reaching steady state is recorded. For each initial elevation, closer control start time to the end of flight costs less convergence time, and for different initial elevations, lower initial elevation angle costs less convergence time.

As seen from the right side figures, no oscillation process is indicated from the deviation between numerical and analytical swerving increment orientations. The deviations are firstly decreased during the beginning of control period and then negative deviations increased, and time durations for the deviations decreasing to less than $10 \mathrm{deg}$. are recorded. Similar law can be seen from the recorded orientation deviation decreasing time of TARI and swerving increment, and the swerving increment orientation deviations are converged in a shorter time than the TARI orientation deviations under the same initial elevation. It is worth noting that, at the start time of $59 \mathrm{sec}$ in Figure 15, the numerical TARI orientation reaches the critical divergence state, and the analytical orientation still 
represents trim value of the oscillation process. Moreover, the analytical and numerical solution of swerving orientations shows little affection by the critical divergence state of TARI.

\section{Conclusions}

This paper focuses on the swerving orientation prediction for the square crossing fixed-cant canard configuration. Analytical expressions of TARI and swerving increment orientation are deduced without the attitude information. Based on the simulations under different initial elevation and control time conditions, the following conclusions can be obtained.

(1) Analytical orientations of TARI and swerving increment indicate the trim value of the numerical orientations. Deviations between numerical and analytical orientations converge in period of time after control started.

(2) For each initial elevation, closer control start times to the end of flight cost less convergence time. For different initial elevations, lower initial elevation costs less convergence time.

(3) Based on the target trajectory angular rate orientation or target swerving orientation provided by navigation system, the desired rolling angle of canard frame can be determined by the reverse solution of increment orientation analytical expressions. By setting appropriate control start time and duration, cost-effective guidance strategy involving no empirical formula for fixed-cant canard configuration will be accomplished.

\section{Appendix}

\section{Force and Moment Expressions}

The direction of drag vector points at the negative $x_{2}$; in the VRF, the expression of $\mathbf{R}_{x}$ can be written as

$$
\mathbf{R}_{x}=\left[\begin{array}{c}
-1 \\
0 \\
0
\end{array}\right] \cdot Q v^{2} c_{x}
$$

Lift vector was obtained by transforming the normal force in the NRRF, and the short form of $\mathbf{R}_{y}$ is

$$
\mathbf{R}_{y}=\left[\begin{array}{c}
\cos \delta_{2} \cos \delta_{1}-1 \\
\cos \delta_{2} \sin \delta_{1} \\
\sin \delta_{2}
\end{array}\right] \cdot Q v^{2} c_{y}^{\prime}
$$

Direction of the Magnus force vector points at the direction of $\mathbf{v} \times \boldsymbol{\xi}$, and the expression of $\mathbf{R}_{z}$ is

$$
\mathbf{R}_{z}=\left[\begin{array}{c}
0 \\
\sin \delta_{2} \\
-\cos \delta_{2} \sin \delta_{1}
\end{array}\right] \cdot Q v^{2} c_{z}^{\prime}
$$

Expression of the gravity vector in the VRF can be written as

$$
\mathbf{G}=\left[\begin{array}{c}
\sin \theta_{a} \cos \psi_{2} \\
\cos \theta_{a} \\
\sin \theta_{a} \sin \psi_{2}
\end{array}\right] \cdot m g
$$

Static moment vector points at the direction of $\mathbf{v} \times \boldsymbol{\xi}$, and the expression of $\mathbf{M}_{z}$ can be obtained as

$$
\mathbf{M}_{z}=\left[\begin{array}{c}
0 \\
-\sin \delta_{1} \sin \beta+\sin \delta_{2} \cos \delta_{1} \cos \beta \\
\sin \delta_{1}
\end{array}\right] \text { Qlv }{ }^{2} m^{\prime} .
$$

Transverse damping moment vector points at the opposite direction of the NRRF spin-rate vector $\omega_{1}$, which can be written as

$$
\mathbf{M}_{z z}=\left[\begin{array}{c}
0 \\
-\omega_{\eta} \\
-\omega_{\zeta}
\end{array}\right] \text { Qldvm }{ }_{z z}^{\prime}
$$

The axial damping moment vector in the NRRF is written as

$$
\mathbf{M}_{x z}=\left[\begin{array}{l}
1 \\
0 \\
0
\end{array}\right] \cdot Q l d v \omega_{\xi} m_{x z}^{\prime}
$$

Magnus moment vector is induced by the Magnus force pointing at the plane of AOA, and $\mathbf{M}_{y}$ points at direction of $\xi \times(\xi \times \mathbf{v})$ and is expressed as

$$
\mathbf{M}_{y}=\left[\begin{array}{c}
0 \\
\sin \delta_{1} \cos \beta+\sin \delta_{2} \cos \delta_{1} \sin \beta \\
\sin \delta_{2} \cos \delta_{1}
\end{array}\right] \text { Qld } \omega_{\xi} v m_{y}^{\prime \prime} .
$$

Canard forces $F_{p d}, F_{c d}$ depending on steering canards and differential canard are expressed as

$$
\begin{aligned}
& F_{p d}=Q v^{2} c_{p d}, \\
& F_{c d}=Q v^{2} c_{c d} .
\end{aligned}
$$




\section{Notations}

\begin{tabular}{|c|c|}
\hline$m:$ & Projectile mass \\
\hline$t:$ & Time \\
\hline$s:$ & Trajectory arc \\
\hline A: & Transverse moment of inertia \\
\hline$C:$ & Projectile axial moment of inertia \\
\hline$l:$ & Reference length \\
\hline$d:$ & Projectile diameter \\
\hline$S:$ & Reference area \\
\hline$g:$ & Acceleration of gravity \\
\hline$X_{c}:$ & $\begin{array}{l}\text { Axial distance between mass center and } \\
\text { bottom end of projectile }\end{array}$ \\
\hline$X_{0}$ : & $\begin{array}{l}\text { Axial distance between mass center and } \\
\text { pressure center of canard frame }\end{array}$ \\
\hline Q: & Dynamic pressure \\
\hline$c_{x}^{\prime}:$ & Projectile drag coefficient derivative \\
\hline$c_{y}^{\prime}:$ & Projectile lift coefficient derivative \\
\hline$c_{z}^{\prime}:$ & Magnus force coefficient derivative \\
\hline$c_{c d}^{\prime}$ & $\begin{array}{l}\text { Differential canard force coefficient } \\
\text { derivative }\end{array}$ \\
\hline$c_{p d}^{\prime}$ & Steering canard force coefficient derivative \\
\hline$m_{z}^{\prime}$ & $\begin{array}{l}\text { Projectile static moment coefficient } \\
\text { derivative }\end{array}$ \\
\hline$m_{z z}^{\prime}:$ & $\begin{array}{l}\text { Transverse damping moment coefficient } \\
\text { derivative }\end{array}$ \\
\hline$m_{x z}^{\prime}:$ & $\begin{array}{l}\text { Projectile roll damping moment } \\
\text { coefficient }\end{array}$ \\
\hline$m_{y}^{\prime \prime}:$ & $\begin{array}{l}\text { Magnus moment coefficient two-order } \\
\text { derivative }\end{array}$ \\
\hline$m_{c d}^{\prime}$ & $\begin{array}{l}\text { Differential canard moment coefficient } \\
\text { derivative }\end{array}$ \\
\hline$m_{p d}^{\prime}:$ & $\begin{array}{l}\text { Steering canard moment coefficient } \\
\text { derivative }\end{array}$ \\
\hline$v:$ & Projectile velocity \\
\hline$\dot{v}:$ & Time derivative of projectile velocity \\
\hline$v^{\prime}:$ & $\begin{array}{l}\text { Trajectory arc derivative of projectile } \\
\text { velocity }\end{array}$ \\
\hline$\theta_{a}, \psi_{2}:$ & Trajectory path/deflection angle \\
\hline$\dot{\theta}_{a}, \dot{\psi}_{2}:$ & Trajectory path/deflection angular rate \\
\hline$\dot{\gamma}:$ & Projectile spin rate \\
\hline$\varphi_{a}, \varphi_{2}:$ & Euler pitch/yaw angle \\
\hline$\omega_{\xi}, \omega_{\eta}, \omega_{\zeta}:$ & Euler angular rate \\
\hline$\delta_{1,2}:$ & Angle of attack \\
\hline$\delta_{1 c, 2 c}:$ & $\begin{array}{l}\text { Angle of attack increment induced by } \\
\text { canard control force }\end{array}$ \\
\hline$\gamma_{c}:$ & Canard rolling angle \\
\hline$F_{x 2}, F_{y 2}, F_{z 2}:$ & Force component in the VRF \\
\hline$M_{\xi}, M_{\eta}, M_{\zeta}$ & Moment component in axis coordinate \\
\hline $\mathbf{R}_{x}:$ & Drag vector \\
\hline $\mathbf{R}_{y}$ : & Lift vector \\
\hline $\mathbf{R}_{z}:$ & Magnus force vector \\
\hline G: & Gravity vector \\
\hline $\mathbf{M}_{x z}:$ & Axial damping moment vector \\
\hline $\mathbf{M}_{z}:$ & Static moment vector \\
\hline $\mathbf{M}_{z z}:$ & Transverse damping moment vector \\
\hline $\mathbf{M}_{y}:$ & Magnus moment vector. \\
\hline
\end{tabular}

\section{Conflict of Interests}

The authors declare that there is no conflict of interests regarding the publication of the paper.

\section{References}

[1] S. Chang, Z. Wang, and T. Liu, "Analysis of spin-rate property for dual-spin-stabilized projectiles with canards," Journal of Spacecraft and Rockets, vol. 51, no. 3, pp. 958-966, 2014.

[2] F. Fresconi and P. Plostins, "Control mechanism strategies for spin-stabilized projectiles," Proceedings of the Institution of Mechanical Engineers, Part G: Journal of Aerospace Engineering, vol. 224, no. 9, pp. 979-991, 2010.

[3] G. Cooper, F. Fresconi, and M. Costello, "Flight stability of asymmetric projectiles with control mechanisms," in Proceedings of the AIAA Atmospheric Flight Mechanics Conference, Toronto, Canada, August 2010.

[4] D. Zhu, S. Tang, J. Guo, and R. Chen, "Flight stability of a dualspin projectile with canards," Proceedings of the Institution of Mechanical Engineers Part G: Journal of Aerospace Engineering, 2014.

[5] S. Theodoulis, V. Gassmann, P. Wernert, L. Dritsas, I. Kitsios, and A. Tzes, "Guidance and control design for a class of spinstabilized fin-controlled projectiles," Journal of Guidance, Control, and Dynamics, vol. 36, no. 2, pp. 517-531, 2013.

[6] F. Sève, S. Theodoulis, P. Wernert, M. Zasadzinski, and M. Boutayeb, "Pitch/Yaw channels control design for a $155 \mathrm{~mm}$ projectile with rotating canards, using a $H_{\infty}$ Loop-Shaping design procedure," in Proceedings of the AIAA Guidance, Navigation, and Control Conference, AIAA 2014-1474, 2014.

[7] E. Gagnon and M. Lauzon, "Course correction fuze concept analysis for in-service $155 \mathrm{~mm}$ spin-stabilized gunnery projectiles," in Proceedings of the AIAA Guidance, Navigation and Control Conference and Exhibit, Honolulu, Hawaii, USA, August 2008.

[8] S. Theodoulis, I. Kitsios, L. Dritsas, A. Tzes, and P. Wernert, "Autopilot strategies of guided projectiles for terminal guidance," in Proceedings of the 19th Mediterranean Conference on Control and Automation (MED '11), pp. 248-253, June 2011.

[9] F. Fresconi, "Guidance and control of a projectile with reduced sensor and actuator requirements," Journal of Guidance, Control, and Dynamics, vol. 34, no. 6, pp. 1757-1766, 2011.

[10] D. Storsved, "PGK and the impact of affordable precision on the fires mission," in Proceedings of 43rd Annual Guns \& Missiles Symposium, DTIC, New Orleans, La, USA, 2008.

[11] J. A. Clancy, T. D. Bybee, and W. A. Friedrich, "Fixed canard 2-D guidance of artillery projectiles," Patent: US 6,981,672 B2.-ZH, USA, 2006.

[12] B. Burchett, A. Peterson, and M. Costello, "Prediction of swerving motion of a dual-spin projectile with lateral pulse jets in atmospheric flight," Mathematical and Computer Modelling, vol. 35, no. 7-8, pp. 821-834, 2002.

[13] D. Ollerenshaw and M. Costello, "Simplified projectile swerve solution for general control inputs," Journal of Guidance, Control, and Dynamics, vol. 31, no. 5, pp. 1259-1265, 2008.

[14] C. H. Murphy, "Gravity- induced angular motion of a spinning missile," Journal of Spacecraft and Rockets, vol. 8, no. 8, pp. 824$828,1971$. 


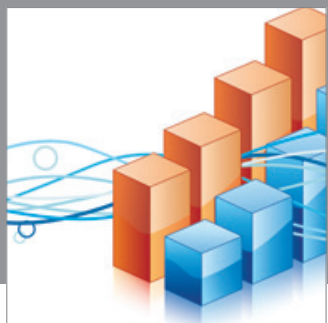

Advances in

Operations Research

mansans

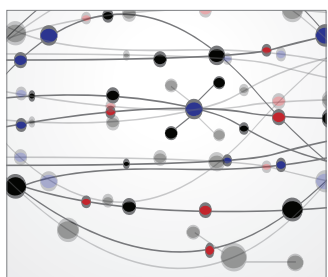

The Scientific World Journal
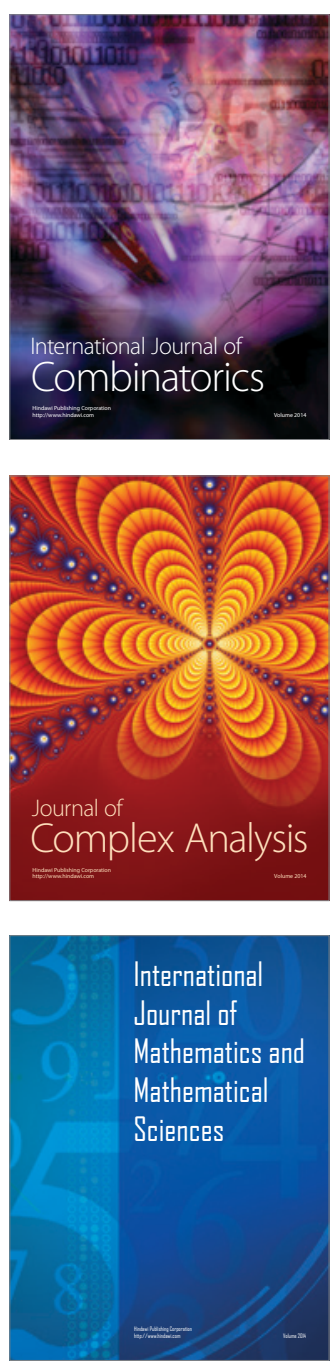
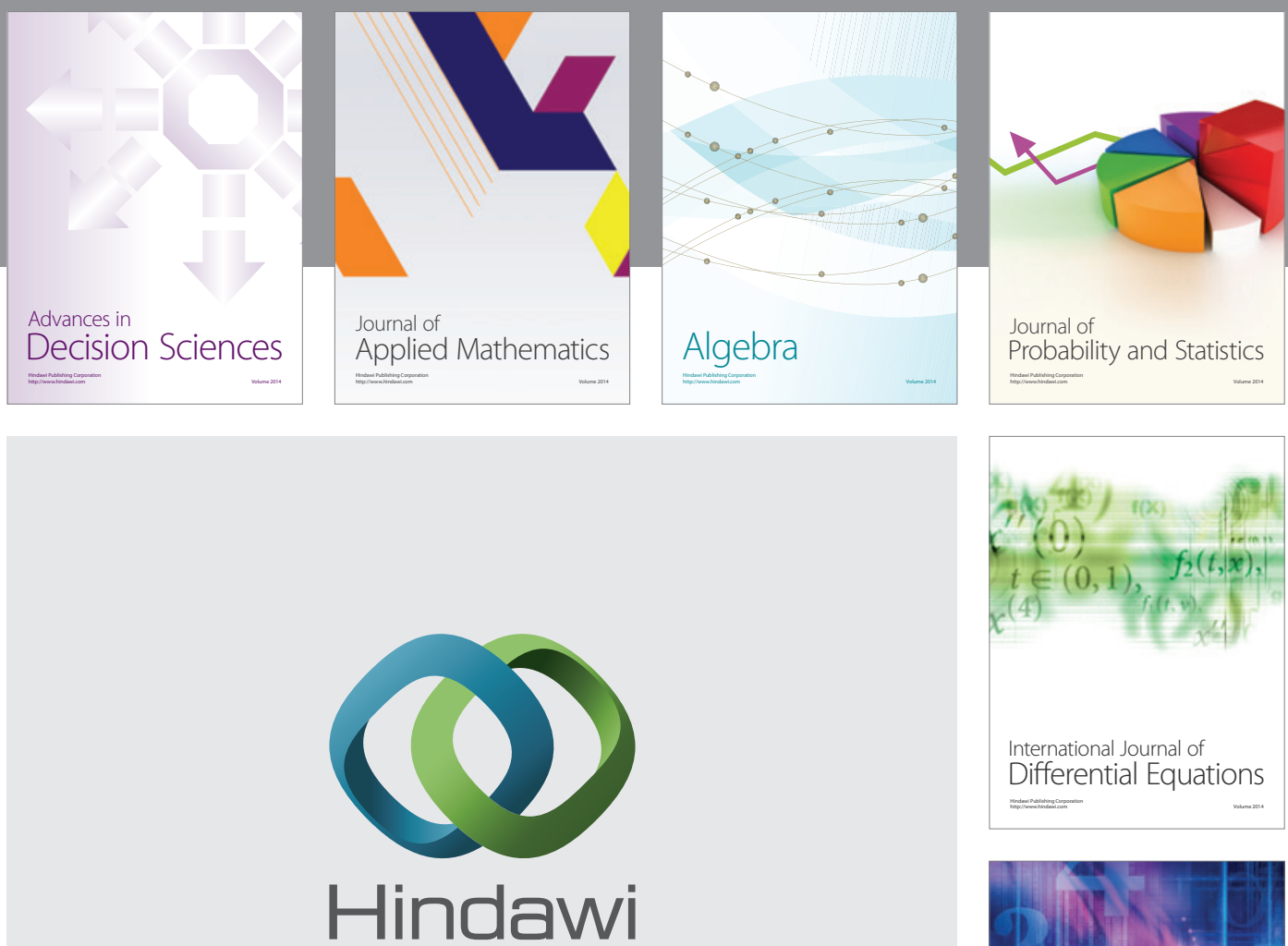

Submit your manuscripts at http://www.hindawi.com
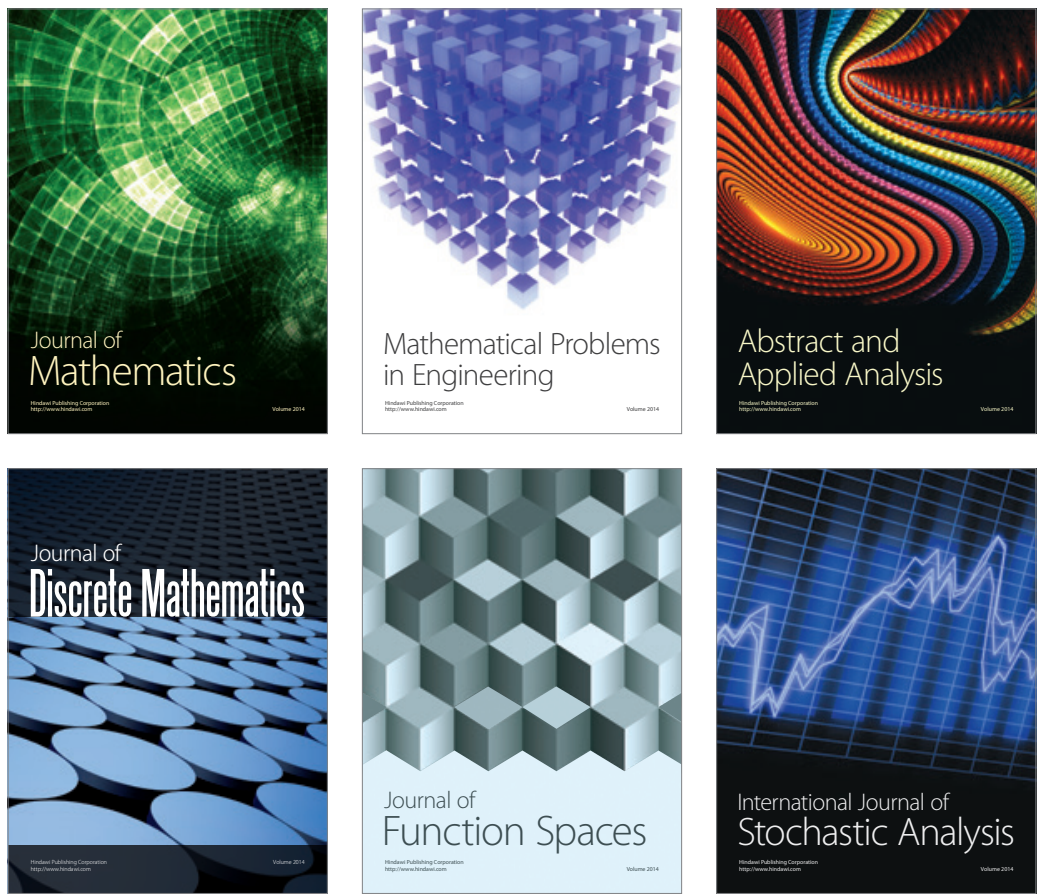

Journal of

Function Spaces

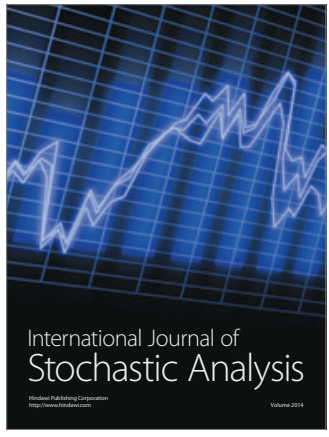

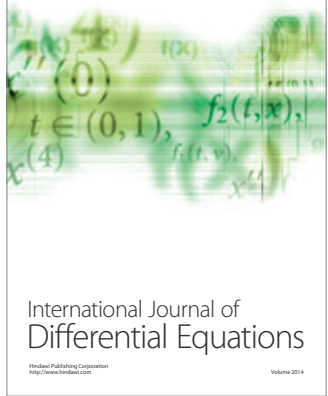
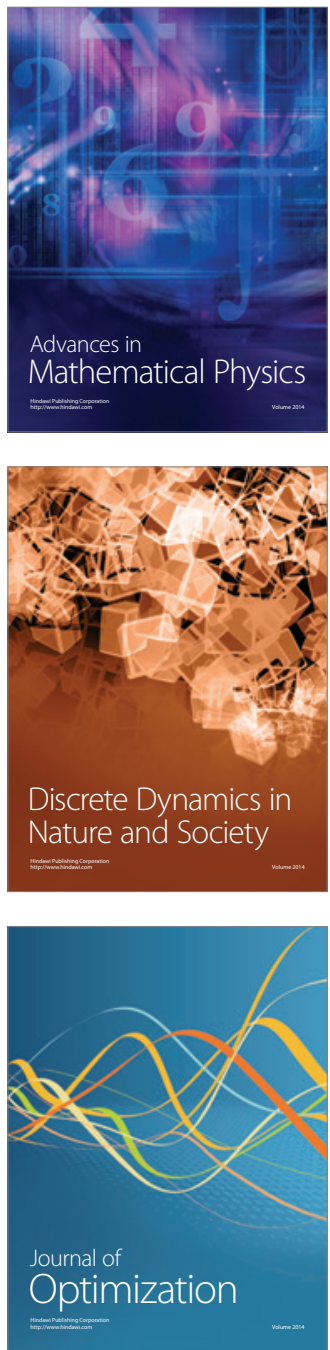